Research Article

\title{
Unloading Rheological Test and Model Research of Hard Rock under Complex Conditions
}

\author{
Longyun Zhang ${ }^{1}$ and Shangyang Yang $\mathbb{D I D}^{2,3}$ \\ ${ }^{1}$ Geotechnical and Structural Engineering Research Centre, Shandong University, Jinan 250061, China \\ ${ }^{2}$ Department of Physics and Mathematics, Shandong Jiaotong University, Jinan 250023, China \\ ${ }^{3}$ Key Laboratory for Health Monitoring and Control of Large Structures, Shijiazhuang 050043, China \\ Correspondence should be addressed to Shangyang Yang; 26916225@qq.com
}

Received 4 December 2019; Revised 31 January 2020; Accepted 28 February 2020; Published 11 May 2020

Academic Editor: Paweł Kłosowski

Copyright ( 92020 Longyun Zhang and Shangyang Yang. This is an open access article distributed under the Creative Commons Attribution License, which permits unrestricted use, distribution, and reproduction in any medium, provided the original work is properly cited.

\begin{abstract}
In order to study the unloading rheological properties of hard rock under complicated conditions, the proposed Mengdigou hydropower station with a granite dam foundation was taken as the research object. This paper studied the unloading rheological properties of the granite by testing means, and it obtained the unloading rheological deformation characteristics and deformation rules of the granite under complex stress conditions. The granite unloading rheological characteristics of the whole stress-strain curve were analyzed, and the nonlinear unloading rheological model (FOD-HKVP) was proposed based on the fractional-order derivative. When certain conditions are satisfied, it is possible to degrade this model into the classical Nishihara model, but it should be noted that the FOD-HKVP model is a more reasonable way to determine the long-term strength of the granite and to consider also the rock unloading rheological damage effect. It introduced the fractional-order viscous body of combination components with a different fractional-order derivative to describe the nonlinear creep characteristics of the granite unloading, which provides an adequate description of steady-state creep and accelerated creep properties. Following the analysis of test data and parameter identification for the FOD-HKVP model, the rationality of the model was validated. Compared with the Nishihara model, the fitting accuracy of FOD-HKVP model was more accurate.
\end{abstract}

\section{Introduction}

The engineering rock mass has a complex geological structure, and rheological properties are one of the most important mechanical characteristics. With the rapid development of the economy, paired with rapid progress in science and technology, many large water resources and hydropower engineering facilities continuously extend deep underground, arriving at points where the geological rock mass is situated in a more complex environment. For many large hydropower stations in China, the buried depth of the foundation engineering of underground caverns is larger, and most of the dam foundation rock mass is comprised of granite, marble, and other hard masses. The natural condition is that rock masses are in a three-dimensional, highstress state, and so cavern excavation has the potential to lead to rock mass unloading. Importantly, this results in changes in the mechanical properties of the rock, thereby affecting the stability and safety of the construction of the underground engineering. As a result, the evolution rules regarding the creep mechanics behaviour of dam slopes under the condition of unloading, as well as research into the constitutive model, have important practical significance for the long-term safety evaluation of high dam projects, ultimately affecting the degree to which the normal operation of hydropower stations can be guaranteed.

Many experimental studies of rock creep characteristics have been conducted by researchers both at home and abroad, with the main reported result being that rock creep is characterised by several stages. These are the following: firstly, the initial creep stage; secondly, the steady-state creep stage; and finally, the accelerated creep stage. With the 
continuous development of rock mechanic tests and research, the literature has gradually found that unloading rock mass mechanics is more in accordance with the actual state of engineering mechanics [1-4]. In view of this, researchers have proposed unloading rock mechanics, which is primarily concerned with the actual mechanical conditions of engineering. Extending this research, based on unloading rock mass mechanics, many scholars have conducted rock unloading experiments in combination with practical engineering projects. Some scholars have obtained the unloading creep characteristics of rocks under different conditions mainly through the tests of rock specimens or rock materials and analyzed the creep deformation rules, creep rate change rules, stress change rules, creep damage characteristic, and long-term strength of rocks in detail according to the test results. For example, in the study conducted by Sorace [5], the researcher implemented the long-term creep test of specimens to investigate the creep properties of rock. This involved several cycles of unloading and loading at the maximum stress value for various stages of steady-state creep, followed by the proposal of a simple mathematical model to describe the test result. Bazhin and Murashkin [6] studied the phenomenon of loading and unloading creep deformation, as well as the stress relaxation characteristics of micropore areas of the material under the condition of hydrostatic pressure. Based on the research, it was possible to establish the nonlinear rheological model, which describes the material's loading and unloading plastic deformation and creep deformation. In the notable study conducted by Zhao [7], the researcher examined the creep properties of intact limestone specimens and fractured specimens by applying the multistage loading and unloading triaxial creep test. The results indicated that fractured rock specimens are associated with a longer creep time, a larger transient creep, and a larger steady-state creep rate. In the abovementioned works, the researchers mainly focused on the creep characteristic of the rock under loading and unloading conditions, while the creep characteristics of rocks under unloading conditions were further studied by later authors. For example, Jiang et al. [8] applied the uniaxial compression and triaxial unloading capacity test, which showed that unloading tests produce greater expansion under identical deviatoric stress. Additionally, Liu et al. [9] considered the excavation involved in unloading rock destruction, and this was followed by an investigation of marble unloading creep properties by implementing triaxial unloading creep tests. The findings indicated that the rock unloading creep rate and creep deformation increase with an increase in axial stress, while they decrease with an increase in confining pressure. Deng et al. [10] examined the grading unloading rheological test in the context of sandy mudstone, and this was paired with the investigation of the unloading creep deformation characteristics and damage characteristics of mudstone under constant axial pressure, unloading confining pressure, and added axial pressure unloading confining pressure for two different paths. In addition, some researchers were dedicated to studying the behavior of creep damage of rock under unloading conditions from microscopics; for instance, Wang et al. [7] conducted ample experiments to show the effect of initial creep damage on unloading failure of rock from macromesoscopic perspective and verified the agreement between the mesoscopic pore parameter and creep damage variable.

Finally, rheological models represent fundamental theoretical approaches that can be mobilised when analysing rock mass rheological mechanical properties. Furthermore, rheological modelling is a crucial tool that can be applied when attempting to examine the implications of rock rheological mechanics theory. In recent years, research in the domain of rock rheological model has advanced considerably, and the experience rheological model, the original combination model, and the nonlinear rheological mechanics model, among others, have been widely used. Among them, the measure of using experimental data inversion on known rheological model parameters, accompanied by the identification of unknown models, has become one of the more commonly used research methods.

However, the current rock mass rheological model theory is still a difficult issue in the study of rock mass mechanics. Many major rock mass engineering projects have highlighted serious problems which underlie the existing research initiatives addressing rock mass rheological model theory. Many scholars use damage mechanics, thermodynamics, fracture mechanics, energy transfer, and the theory of diffusion for model analysis, and this has underpinned the acquisition of relatively satisfactory results. For example, based on the theory of fractional calculus, Zhou et al. [12] constructed a viscosity elastic-plastic constitutive model to describe the creep property characteristics of halite. Additionally, based on soft rock, argillaceous siltstone, constant axial compression, and graded and unloading confining pressure test results, Wang et al. [13] constructed the nonlinear damage rheological model. Nedjar and Roy [14] established the three-dimensional mechanical model on the basis of viscoelastic continuum damage mechanics, which enabled accurate descriptions of the long-term creep behaviour of gypsum rock. This was followed by the construction of a constitutive model that can describe the entire creep process of granite under different temperature conditions. In another noteworthy research project, Chen and Konietzky [15] established a discrete element method, relying on a numerical model based on heterogeneous particles, the purpose of which was to describe the three-phase aging properties of rock creep. Furthermore, Lu et al. [16] proposed a dual-scale model to simulate the creep aging damage, deformation, and fracture behaviour of heterogeneous brittle rock. Wu et al. [17] established an improved Maxwell creep model based on the theory of fractional calculus, and it was successful in providing a more effective description of the creep properties of rock masses. In addition, based on an examination of the relationship between the macro- and the micromechanics definitions of rock creep damage, $\mathrm{Li}$ and Shao [18] established a micromechanics-based model. This was then used to study the creep properties of rocks under variable stress paths. By adopting the complex deformation mechanism of the creep model, the valuable research conducted by Firme et al. [19] provided an accurate description of the sticky elastoplastic creep characteristics of soft rock and then applied the finite element [11] numerical simulation to the creep test. Recently, 
Tang et al. [20] proposed a four-element creep model based on variable fractional derivatives and continuous damage mechanics, which was used to simulate the creep behavior of rock piecewise. Hu et al. [21] carried out creep test to figure out the creep rule and mechanical property of fractured phyllite and modified Burger's model to analyze the creep characteristics of fractured phyllite. Wang et al. [22] carried out the triaxial unloading creep tests of layered rock specimen of Jinping II hydropower station and acquired the creep deformation curves under different confining pressures. Through analysing the creep parameters, a nonlinear creep damage model of rock under unloading was proposed to reflect the nonlinear characteristics of rock creep.

From what have been discussed above, some of the models were established by the combination of elastic elements, plastic elements, or elastoplastic elements, some were established by modifying the classical mechanical models, and others were established by introducing new parameters. Additionally, some models with few parameters and good applicability were mostly applicable to soft rocks; despite the wealth of literature addressing related topics, it is important to recognize that the available evidence pertaining to the unloading rheological study of hard rock masses is insufficient. One of the main reasons for this relates to the limitations of testing equipment and testing conditions, especially regarding the unloading rheological test for hard rock and its constitutive model. Therefore, with respect to the hard rock unloading rheological test and the theoretical research, further areas for exploration are numerous. In view of this, the purpose of the present research was to apply the hard rock unloading rheological test to the proposed Mengdigou hydropower station, thus informing the present state of research regarding unloading rock mass rheological studies. Here, it is particularly necessary to introduce the mengdigou hydropower station, which locates in Sichuan, China. It is a concrete hyperbolic arch dam, with the maximum dam height of $240 \mathrm{~m}$, light-colored granite is mainly developed in the dam foundation, and the mechanical properties of granite have influenced greatly under the action of excavation and unloading during the long construction period that will last 8.1 years.

According to the unloading rheological properties of hard rock, this paper capitalised on the theory of fractional calculus, and it constructed the constitutive model to describe the rheological deformation characteristics of hard rock unloading. Noteworthily, the model was verified. The fact that the publication of this result, as well as related results in the literature, has promoted and enriched a new way of thinking about hard rock unloading rheological mechanics research should not be overlooked. Furthermore, it has provided experimental and theoretical support for the water resources and hydropower dam project, all the while aiding in the long-term stability of other comparable engineering initiatives.

\section{Hard Rock Unloading Rheological Test and Analysis}

2.1. Unloading Rheological Test. In order to study the unloading rheological characteristics of hard rock, the granite of Mengdigou hydropower station was taken as the main research object. According to the actual engineering situation, the unloading rheological test programme was designed as keeping the axial stress $\left(\sigma_{1}\right)$ unchanged, removing the confining pressure step by step. Then, the unloading rheological tests of granite were carried out. All the granite samples used in the tests were taken from the dam foundation of Mengdigou hydropower station, and the rock samples for testing were cut into $\phi 50 \mathrm{~mm} \times 100 \mathrm{~mm}$ standard cylinder specimens. The rock automatic triaxial servo rheometer (RLW-1000 kN) was used in this test, which was independently researched and developed by Shandong University. The purpose of the instrument is to conduct the unloading rheological experimental study on the granite from the Mengdigou hydropower station.

Based on the conventional triaxial test of hard rock, we found that the peak intensity of hard rock is higher, while the bearing capacity is larger. With the engineering practice of higher stress on a high dam slope, it is necessary when making unloading rheological test plans to take into consideration the larger initial confining pressure and the higher-level axial stress. These are pertinent when conducting the unloading rheological experiment on the basis of the axial stress $\sigma_{1}$, which remains unchanged, and step-bystep unloading confining pressure. The initial axial stress level is generally $40-50 \%$ of the peak intensity. Furthermore, owing to the high strength of hard rock, the creep deformation for time dependence is stronger, and so in order to observe more obvious creep deformation, unloading rheological length was set at each level for 50-70 h. Additionally, this enabled the study to satisfy the accuracy requirements of unloading rheological levels, and the unloading step was set at 5-8. For the purpose of guaranteeing the smoothness of the test, the classification of unloading at a constant discharge rate was $100 \mathrm{~N} / \mathrm{s}$. The test path is shown in Figure 1, while the test process is outlined in Figure 2, where $\sigma_{1}$ represents the axial stress; $\sigma_{3}$ represents the confining pressure; and $\left(\sigma_{1}-\sigma_{3}\right)$ represents the axial deviatoric stress.

The conventional triaxial compression stress-strain curve of granite under different confining pressures is shown in Figure 3. The cohesive force of granite was $c=21.30 \mathrm{Mpa}$, while the internal friction angle was $\phi=54.25^{\circ}$.

2.2. Unloading Rheological Test Analysis. Based on the results of the unloading rheological test, the rock unloading rheological stress-strain curve was plotted. Drawing on the confining pressure of $40 \mathrm{MPa}$ as an example owing to the limited length, Figure 4 presents the unloading rheological stress-strain curve of the granite sample under the confining pressure of $40 \mathrm{MPa}$, while Figures 5 and 6 illustrate the macroscopic and microscopic rock damage modes, where $\varepsilon_{1}$ presents the axial strain of granite, $\varepsilon_{3}$ presents the lateral strain of granite, $\varepsilon_{v}$ presents the volume strain of granite, and $\left(\sigma_{1}-\sigma_{3}\right)$ presents the deviatoric stress.

By analysing the rock unloading creep curve characteristics of Figures 2-6 for the unloading rheological test damage characteristics of the rock, it is possible to draw the following conclusions: 


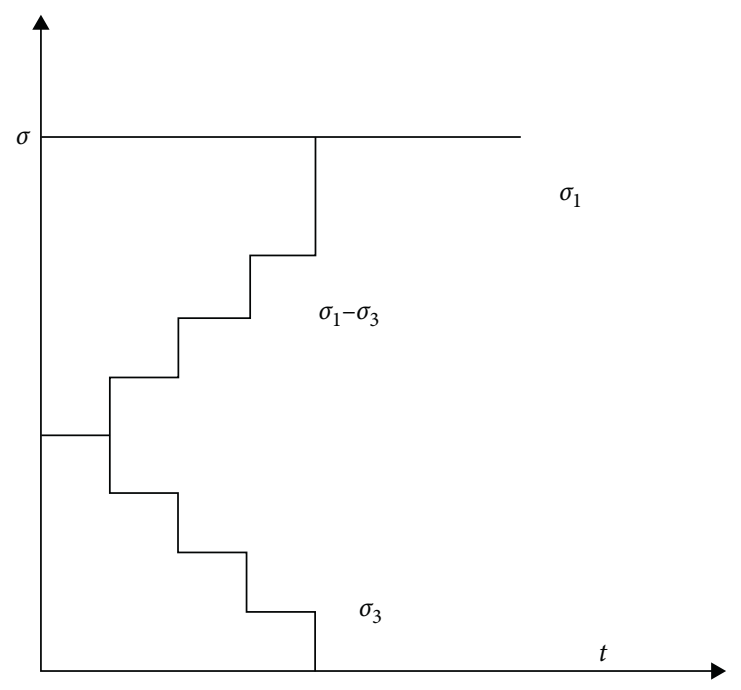

FIgURE 1: Unloading path diagram.

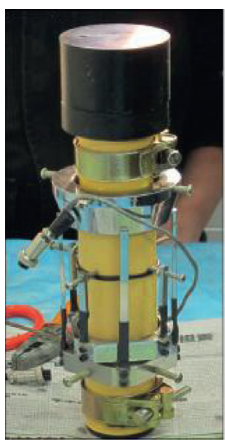

(a)

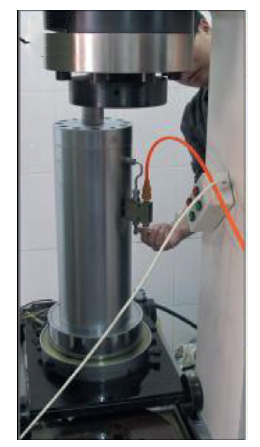

(d)

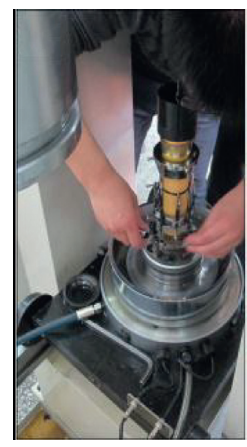

(b)

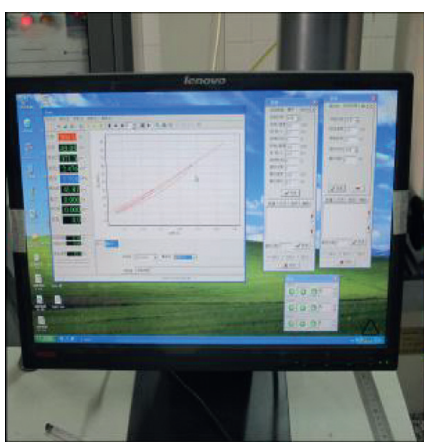

(e)

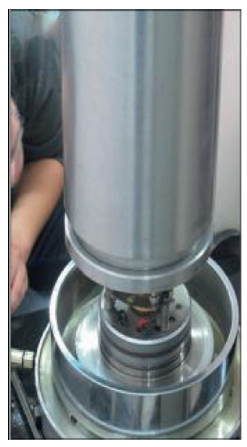

(c)

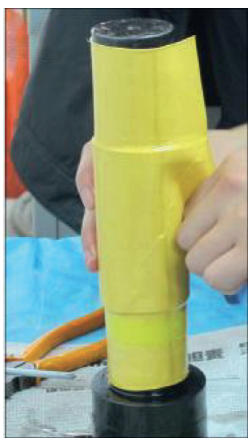

(f)

Figure 2: Operation process for the tests. (a) Specimen packaging. (b) Specimen installation. (c) Closed pressure chamber. (d) Oil filled. (e) Control system operation. (f) Specimen demolition.

(1) Under various stress levels, the unloading moment will produce instantaneous elastic strain.

(2) Rock flowing deformation exists when unloading, and there also exists a rheological threshold. When the stress level exceeds the rheological threshold, even if the stress level remains the same, aging deformation will occur.

(3) When the stress level is low, rock creep strain over time facilitates a slowdown in growth, characterised by slow creep properties. On the contrary, when the stress level is higher, the rock creep strain shows a steady growth in speed over time, and it is characterised by stable creep properties. In addition, when the stress level has reached the long-term strength of the rock, rock creep strain will accelerate over time, characterised by the accelerated creep property. Finally, the rock will undergo dilatancy damage, facilitating a rapid increase in deformation, characterised by brittle failure. 


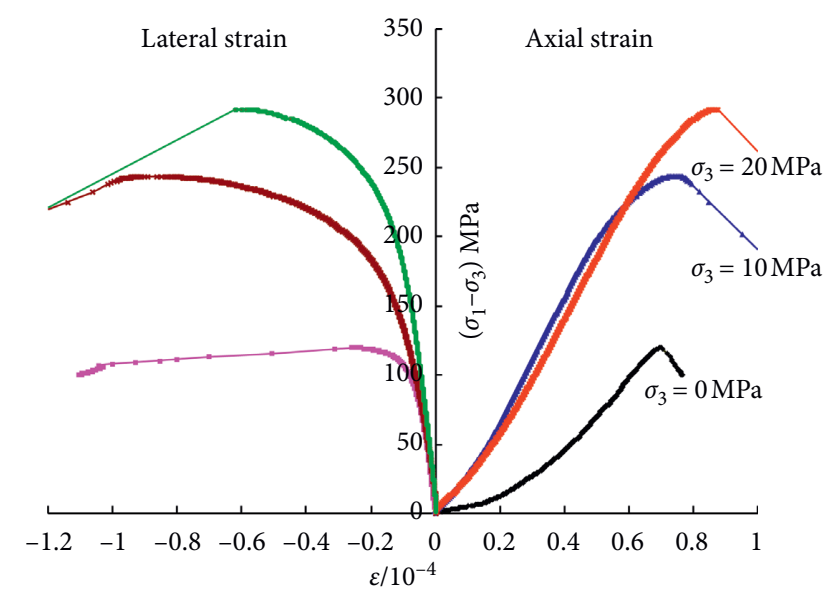

Figure 3: Triaxial compression stress $\left(\sigma_{1}-\sigma_{3}\right)$-strain $(\varepsilon)$ curve for granite under different confining pressures.

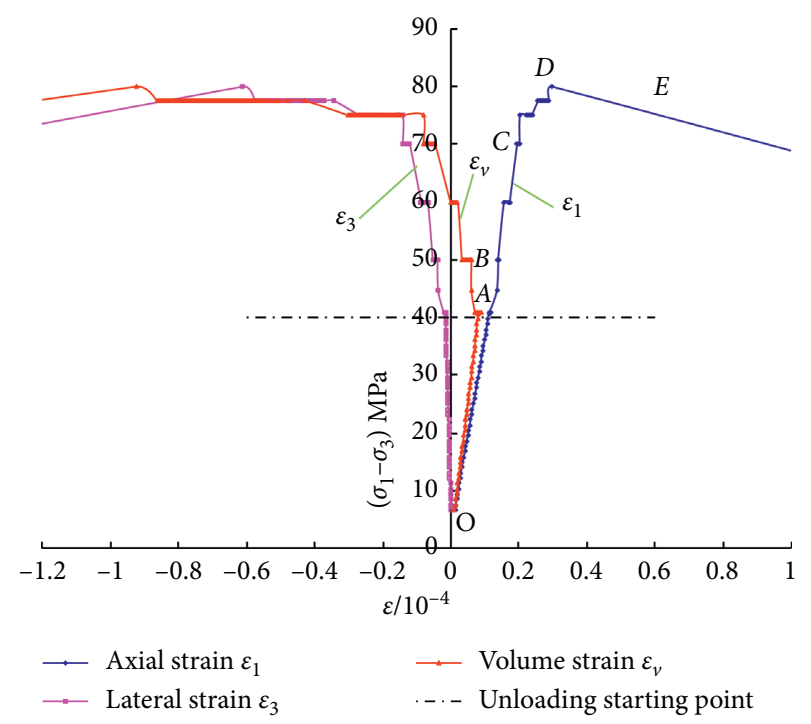

FIGURE 4: Relationship between deviatoric stress $\left(\sigma_{1}-\sigma_{3}\right)$ and strain $(\varepsilon)$.

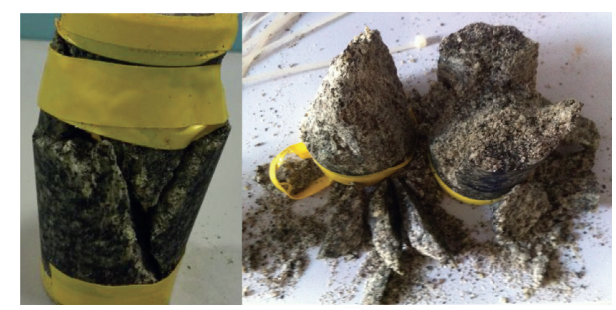

Figure 5: Macroscopic damage mode of granite.

(4) The microscopic damage forms of the rock show that damage occurred during the unloading rheological process.

The analysis of the characteristics of the hard rock unloading creep test curve indicates that the creep deformation of rock unloading involves instantaneous deformation, slow creep deformation, steady-state creep deformation, and acceleration creep deformation. Each deformation stage presents distinctive characteristics. When the stress level is lower than long-term strength, hard rock displays typical viscoelastic characteristics; however, when the stress level is greater than the long-term strength and reaches the fracture stress level, it displays typical sticky plastic characteristics.

\section{Modelling Problems That Must Be Solved}

According to the unloading rheological test results, the construction of a hard rock unloading creep model should include an elastic element, viscous components, and plastic components. This is because these components can describe the damage characteristics of hard rock unloading. Therefore, the following four problems need to be solved: (1) reasonably determining the long-term strength of rock; (2) identifying different degrees of damage at each unloading creep stage; (3) ensuring that the steady-state creep stage unloading element model reflects smooth deformation characteristics; and (4) ensuring that the unloading accelerated creep phase component model clearly describes the unloading rheological characteristic of more apparent dilatancy.

The intention of this research is to draw on the following methods to solve these problems. Firstly, the creep rate intersection method is an effective measure that can be used to analyze the long-term strength of hard rock unloading and also to derive a reasonable long-term strength threshold; secondly, through the analysis of the characteristics of the rock damage, it will be appropriate to define damage variables and establish the parameters damage through the evolution equation, and through the damage evolution of model parameters at different stages of creep, to reflect the damage effect of rock during different unloading phases; and finally, to build the fractional unloading rheological element, with this process based on the change of the fractional-order derivative-order time. Regarding the last point, this approach will not only reflect the smooth deformation characteristics of the unloading steady-state creep stage, but it will also ensure more apparent dilatancy for the unloading accelerated creep.

3.1. Creep Rate Intersection Method Analysis regarding LongTerm Strength. Through the analysis of the steady-state creep properties, it is possible to find that for sample in the steady-state creep stage, there must be a node in its axial creep rate and the lateral creep rate curve, which is the critical point of rock creep damage. This node corresponds to the strength, which is the long-term strength of the rock. As outlined in Figure 7, this is referred to as the steady-state creep rate intersection method.

Based on the analysis of the test results from the unloading rheological test, the rock sample became unstable after volume expansion. Therefore, the reliable values of the long-term strength shall be at the volume strain compression stage, namely, the $\varepsilon_{v}>0$ unloading creep stage. In addition, the point of intersection for the unloading creep rate-stress curve (CR- $\sigma$ ) becomes the first target of the ideal long-term strength. It should be noted that, firstly, the intersection 

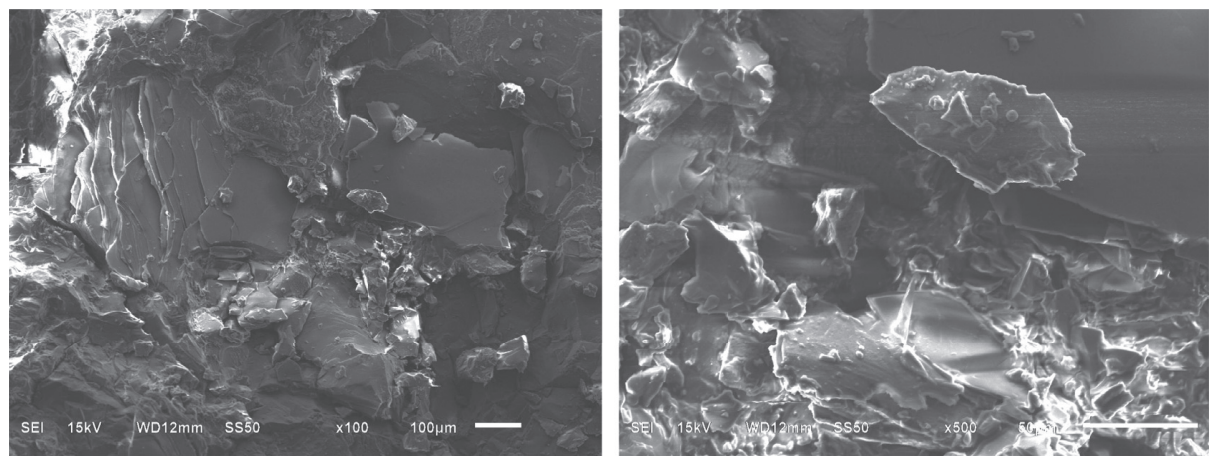

Figure 6: Microscopic damage mode of granite.

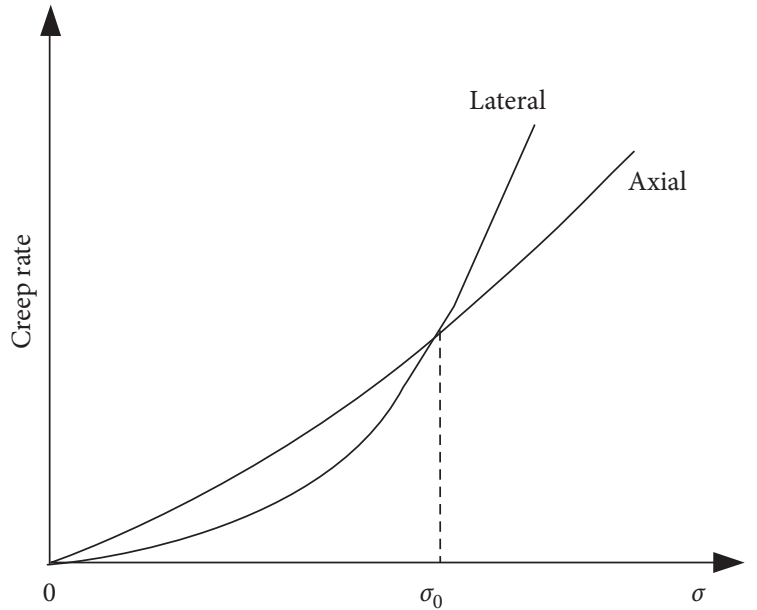

FIGURE 7: Schematic diagram of "intersection point of creep rate curve method."

point meets the $\varepsilon_{v}>0$ unloading creep stage. And secondly, the analysis shows that when stress level exceeds this stress value, the rock enters an expanding and inflating stage. Therefore, it is usually reasonable to take this point as the long-term strength threshold, below which the rock can maintain its long-term stability. In the event that the stress level exceeds the critical value, the rock will transition from the steady creep stage to the accelerated creep stage, and damage will be sustained rapidly.

3.2. Analysis of Fracture Characteristics. The ultrasonic flaw detection (UFD) test is regarded as one of the most effective ways to study the evolution of internal structural rock damage. It relies on the longitudinal wave velocity of the rock rheological ultrasonic test. In this context, it is possible to define the damage variable [23] in the following way:

$$
D=1-\frac{1}{1+\varepsilon_{v}} \frac{v_{p}}{v_{0}},
$$

where $\varepsilon_{v}$ represents the volumetric strain, $v_{p}$ represents the longitudinal wave velocity, and $v_{0}$ represents the initial wave velocity.

Equation (1) can be further simplified to yield the following:

$$
D=1-e^{-\alpha t}
$$

where $\alpha$ is the related coefficient of longitudinal wave velocity, the initial wave velocity, and the volumetric strain of rock material. Here, we call it as damage parameter.

Hence, the damage evolution equation can be formulated as follows:

$$
\left\{\begin{array}{l}
\eta_{1}^{\beta^{\prime}}(t)=(1-D) \eta_{1}^{\beta}=e^{-\alpha t} \eta_{1}^{\beta}, \\
\eta_{1}^{\gamma^{\prime}}(t)=(1-D) \eta_{1}^{\gamma}=e^{-\alpha t} \eta_{1}^{\gamma}, \\
E_{0}^{\prime}(t)=(1-D) E_{0}=e^{-\alpha t} E_{0}, \\
E_{1}^{\prime}(t)=(1-D) E_{1}=e^{-\alpha t} E_{1} .
\end{array}\right.
$$

3.3. Unloading Damage Rheology Element Analysis. Generally, complex viscoelastic materials represent the middle ground between the ideal elastic and viscous materials. This stems from the fact that they are characterised by memory characteristics regarding stress and change of strain. Noteworthily, Hooke's law of the elastic solid and Newton's law of the viscous fluid cannot accurately describe the characteristics. In view of this, the fractional-order derivative can be used to describe the complicated physical and mechanical process, greatly overcoming the shortcomings of ineffective inosculation between the theoretical and experimental results [23-25].

3.4. The Fractional-Order Viscous Body. Hooke's law indicates that the constitutive relation for ideal elastic solid is $\sigma(t) \sim \mathrm{d}^{0} \varepsilon(t) / \mathrm{d} t^{0}$, while Newton's law indicates that the constitutive relation for the ideally viscous fluid is $\sigma(t) \sim \mathrm{d}^{1} \varepsilon(t) / \mathrm{d} t^{1}$. In view of this, there is sufficient reason to think that the constitutive relation $\sigma(t) \sim \mathrm{d}^{\beta} \varepsilon(t) / \mathrm{d} t^{\beta}(0 \leq$ $\beta \leq 1)$ coincides with the mechanical property of the viscoelastic body between ideal elastic solids and a fluid with ideal viscosity. $\beta$ takes a boundary value to describe the two viscous and elastic limit states, and when $\beta=1$, the element represents an ideal fluid; however, when $\beta=0$, the element represents the ideal solid. In addition, when $0<\beta<1$, the element represents some state of the object between the ideal fluid and solid state, which is referred to as a fractional viscous body. This is indicated in Figure 8. 


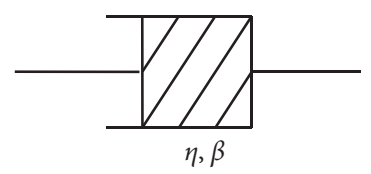

Figure 8: The fractional-order derivative (FOD) viscous body model.

The constitutive relation for the fractional-order viscous body components is as follows:

$$
\sigma(t)=\frac{\eta \mathrm{d}^{\beta} \mathcal{\varepsilon}(t)}{\mathrm{d} t^{\beta}}, \quad 0<\beta<1,
$$

where $\eta$ is the fractional order viscous coefficient, the physical dimension of which is [stress.time $\beta$ ], and $\beta$ is the fractional-order derivative times of the fractional-order viscous body.

When the stress remains unchanged (namely, when $\sigma(t)=$ const), the fractional-order viscous body components will describe the rheological behaviour of material creep. Based on Riemann-Liouville fractional calculus operator theory, addressing the fractional integrals on the left- and right-hand sides of (4) can yield the creep equation of fractional viscous body in the following way:

$$
\varepsilon(t)=\frac{\sigma}{\eta} \frac{t^{\beta}}{\Gamma(1+\beta)}, \quad 0<\beta<1 .
$$

Noteworthily, when the strain remains the same (namely, when $\varepsilon(t)=$ const), the fractional viscous body will describe the rheological behaviour of the material's stress relaxation. Thus, drawing on (5), it is possible to derive the following viscous stress relaxation equation:

$$
\sigma(t)=\eta \varepsilon \frac{t^{-\beta}}{\Gamma(1-\beta)}, \quad 0<\beta<1 .
$$

As outlined in the above equation, the fractional viscous body for different rock material properties, by addressing the change in the fractional-order derivative-order $\beta$ value, can effectively reflect the nonlinear gradients of the material creep process and the gradient material stress relaxation process.

\section{Modelling and Solving the Unloading Nonlinear Rheological Constitutive Model Based on the Fractional-Order Derivative}

To solve the four key problems associated with constructing an unloading rheological model, it is useful to draw on the fractional-order derivative hard rock unloading nonlinear rheological model (FOD-HKVP), as shown in Figure 9. Based on the classical Nishihara model, the key improvements that must be made are as follows: firstly, the whole process of the unloading rheological model must consider the damage of the rock material degradation effect; secondly, the new long-term strength analysis method should be utilised, which is more in line with the hard rock mass unloading rheological phenomenon; and finally, the theory of fractional-order calculus should be introduced.
Regarding the final point, this is noteworthy because the introduction of fractional-order calculus enables us to more effectively reflect the nonlinear creep characteristics of hard rock. In particular, this could reflect the dilation of the accelerating creep phase, as well as the characteristics of brittle failure.

4.1. Stress-Strain Relationship for the Injury Elastomer $(H)$. According to Hooke's law, the creep equation for the injury elastomer is as follows:

$$
\varepsilon_{e}(t)=\frac{\sigma}{E_{0}(1-D)}=\frac{\sigma}{E_{0} e^{-\alpha t}}
$$

where $E_{0}$ is the elasticity modulus of $H$.

4.2. Fractional Damage Viscoelastic Body (KV) Stress-Strain Relationship.

$$
\sigma=\eta_{1}^{\beta} e^{-\alpha t} \frac{\mathrm{d}^{\beta}\left[\varepsilon_{v e}(t)\right]}{\mathrm{d} t^{\beta}}+E e^{-\alpha t} \varepsilon_{v e}(t) .
$$

Namely,

$$
\frac{\mathrm{d}^{\beta}\left[\varepsilon_{v e}(t)\right]}{\mathrm{d} t^{\beta}}=\frac{\sigma}{\eta_{1}^{\beta}} e^{\alpha t}-\frac{E}{\eta_{1}^{\beta}} \varepsilon_{v e}(t) .
$$

Using the fractional-order differential equation to solve (9) will yield an expression for the creep equation of the fractional-order damage viscoelastic body as follows:

$$
\varepsilon_{v e}(t)=\frac{\sigma}{\eta_{1}^{\beta}} e^{\alpha t} \int_{0}^{t} e^{-\alpha \tau} \tau^{\beta-1} \sum_{k=0}^{\infty} \frac{\left(E_{1} / \eta_{1}^{\beta}\right)^{k} \tau^{\beta k}}{\Gamma(k \beta+\beta)} \mathrm{d} \tau,
$$

where $\eta_{1}^{\beta}$ is the viscosity coefficient of fractional damage for a viscoelastic body, and where $E_{\beta, \beta}\left[\left(-\left(E_{1} / \eta_{1}^{\beta}\right)\right) \tau^{\beta}\right]=$ $\sum_{k=0}^{\infty}\left(\left(E_{1} / \eta_{1}^{\beta}\right)^{k} \tau^{\beta k} / \Gamma(k \beta+\beta)\right)$ refers to the two-parameter Mittag-Leffler function.

4.3. The Stress and Strain Relation of the Fractional Damage Viscoplasticity Body (P).

$$
\sigma=\eta_{2}^{\gamma} e^{-\alpha t} \frac{\mathrm{d}^{\gamma}\left[\varepsilon_{v p}(t)\right]}{\mathrm{d} t \gamma}+\sigma_{s}
$$

Namely,

$$
\frac{\mathrm{d}^{\gamma}\left[\varepsilon_{v p}(t)\right]}{\mathrm{d} t \gamma}=\frac{\sigma-\sigma_{s}}{\eta_{2}^{\gamma}} e^{\alpha t}
$$

Using (2)-(4), the Laplace transform and inverse transformation can be performed. Consequently, the creep equation of the viscous plastic body can be obtained:

$$
\varepsilon_{v p}=\left\{\begin{array}{l}
0, \quad \sigma<\sigma_{s}, \\
\frac{\sigma-\sigma_{s}}{\eta_{2}^{\gamma}} t^{\gamma} \sum_{k=0}^{\infty} \frac{(\alpha t)^{k}}{\Gamma(k+1+\gamma)}, \quad \sigma \geq \sigma_{s} .
\end{array}\right.
$$

According to the series-parallel property of the model elements, the total strain of the FOD-HKVP model can be expressed in the following way: 


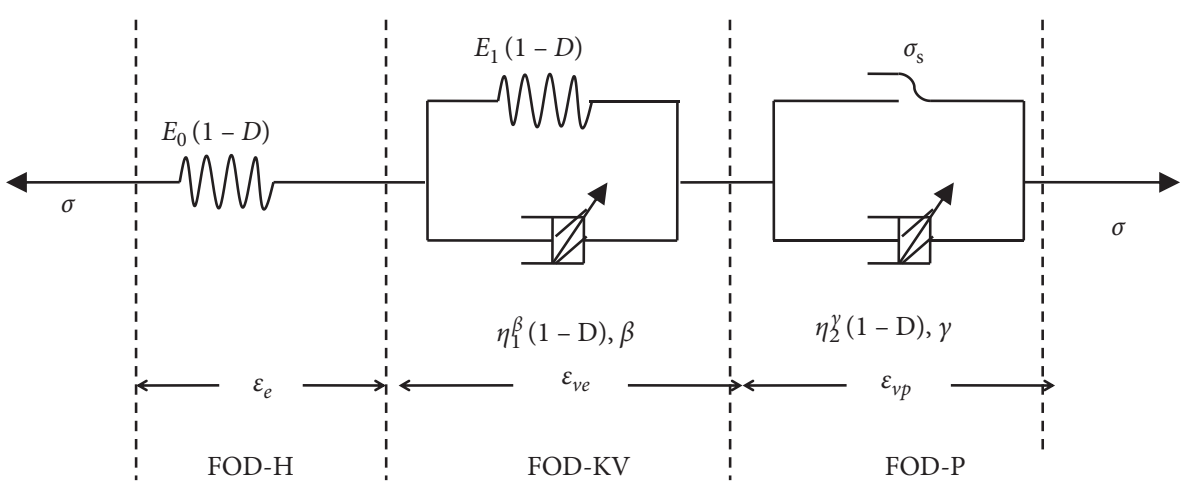

FIGURE 9: Nonlinear unloading rheological constitutive model based on the fractional-order derivative.

$$
\varepsilon(t)=\varepsilon_{e}(t)+\varepsilon_{v e}(t)+\varepsilon_{v p}(t)
$$

By substituting (3), (7), and (10) into (14), it is possible to derive the constitutive equation of the nonlinear rheological model (FOD-HKVP model) based on the fractional order:

$$
\left\{\begin{array}{l}
\varepsilon(t)=\frac{\sigma}{E_{0} e^{-\alpha t}}+\frac{\sigma}{\eta_{1}^{\beta}} e^{\alpha t} \int_{0}^{t} e^{-\alpha \tau} \tau^{\beta-1} \sum_{k=0}^{\infty} \frac{\left(E_{1} / \eta_{1}^{\beta}\right)^{k} \tau^{\beta k}}{\Gamma(k \beta+\beta)} \mathrm{d} \tau, \quad \sigma<\sigma_{s}, \\
\varepsilon(t)=\frac{\sigma}{E_{0} e^{-\alpha t}}+\frac{\sigma}{\eta_{1}^{\beta}} e^{\alpha t} \int_{0}^{t} e^{-\alpha \tau} \tau^{\beta-1} \sum_{k=0}^{\infty} \frac{\left(E_{1} / \eta_{1}^{\beta}\right)^{k} \tau^{\beta k}}{\Gamma(k \beta+\beta)} \mathrm{d} \tau+\frac{\sigma-\sigma_{s}}{\eta_{2}^{\gamma}} t^{\gamma} \sum_{k=0}^{\infty} \frac{(\alpha t)^{k}}{\Gamma(k+1+\gamma)}, \quad \sigma \geq \sigma_{s} .
\end{array}\right.
$$

It is not difficult to see that when $\alpha=0, \beta=1$, and $\gamma=1$, the model degenerates as follows:

$$
\left\{\begin{array}{l}
\varepsilon(t)=\frac{\sigma}{E_{0}}+\frac{\sigma}{E_{1}}\left(1-e^{-\left(E_{1} / \eta_{1}\right) t}\right), \quad \sigma<\sigma_{s}, \\
\varepsilon(t)=\frac{\sigma}{E_{0}}+\frac{\sigma}{E_{1}}\left(1-e^{-\left(E_{1} / \eta_{1}\right) t}\right)+\frac{\sigma-\sigma_{s}}{\eta_{2}} t, \quad \sigma \geq \sigma_{s} .
\end{array}\right.
$$

Noteworthily, (16) is entirely consistent with the classical Nishihara model. Therefore, it is clear that the classical Nishihara model is a particular instance of the FOD-HKVP model when $\alpha=0, \beta=1$, and $\gamma=1$.

\section{Model Verification}

It should first be noted that the parameters $E_{0}, E_{1}, \eta_{1}^{\beta}, \eta^{\gamma}$, and $\alpha$ are contained in the hard rock unloading nonlinear rheological model. Equation (15), namely, the creep equation of FOD-HKVP model, was used to preliminarily fit the creep test curve of hard rock unloading, and during the attenuation creep and steady-state creep stages, $\alpha$ is approximately $0.00001 \mathrm{~h}^{-1}$. Contrastingly, during the accelerating creep stage, $\alpha$ amounts to around $0.00005 \mathrm{~h}^{-1}$. This phenomenon is consistent with the experimental conclusion that the degree of deterioration in the accelerated creep stage is greater than that of attenuation creep and steady-state creep. Therefore, in order to simplify the calculation, $\alpha$ takes the value of $0.00001 \mathrm{~h}^{-1}$ when the FOD-HKVP model is distinguishing (note that the stress level is lower than the long-term strength), while it alternatively takes the value of $0.00005 \mathrm{~h}^{-1}$ (note that the stress level is higher than the longterm strength). Furthermore, the parameters $E_{0}, E_{1}, \eta_{1}^{\beta}, \eta_{2}^{\gamma}$, $\beta$, and $\gamma$ can be identified using the objective function method [13].

To verify the validity of the unloading rheological model (FOD-HKVP), it is useful to make use of the compiled calculation procedure to carry out the finite element analysis and then compare with the experimental data.

The numerical model size is $\phi 50 \mathrm{~mm} \times 100 \mathrm{~mm}$ standard cylinder specimens, a total of 8000 units and 8421 nodes. The model is constrained in the $Z$-direction at the bottom, as shown in Figure 10.

According to the results of unloading rheological test, the creep rate node method was used to determine the longterm strength of rock, which is $62.6 \mathrm{MPa}$. And the damage parameter $\alpha=0.00001 \mathrm{~h}^{-1}$, when $\sigma<62.6 \mathrm{MPa}$, while $\alpha=0.00005 \mathrm{~h}^{-1}$, when $\geq 62.6 \mathrm{MPa}$, and Poisson's ratio was assumed to be a fixed value, which is 0.2 . The axial creep parameter $\sigma$ identification results of the granite under the initial unloading confining pressure of $40 \mathrm{MPa}$ are shown in Table 1, and the model theory curve and test curve are outlined in Figure 11.

Table 1 shows the model parameter identification results, and under identical unloading confiding pressure conditions with varying stress levels, the model's parameters are different. Furthermore, each level of stress is characterised by a distinct set of parameter values. In view of this, we now encounter a form of nonlinear rheology. 


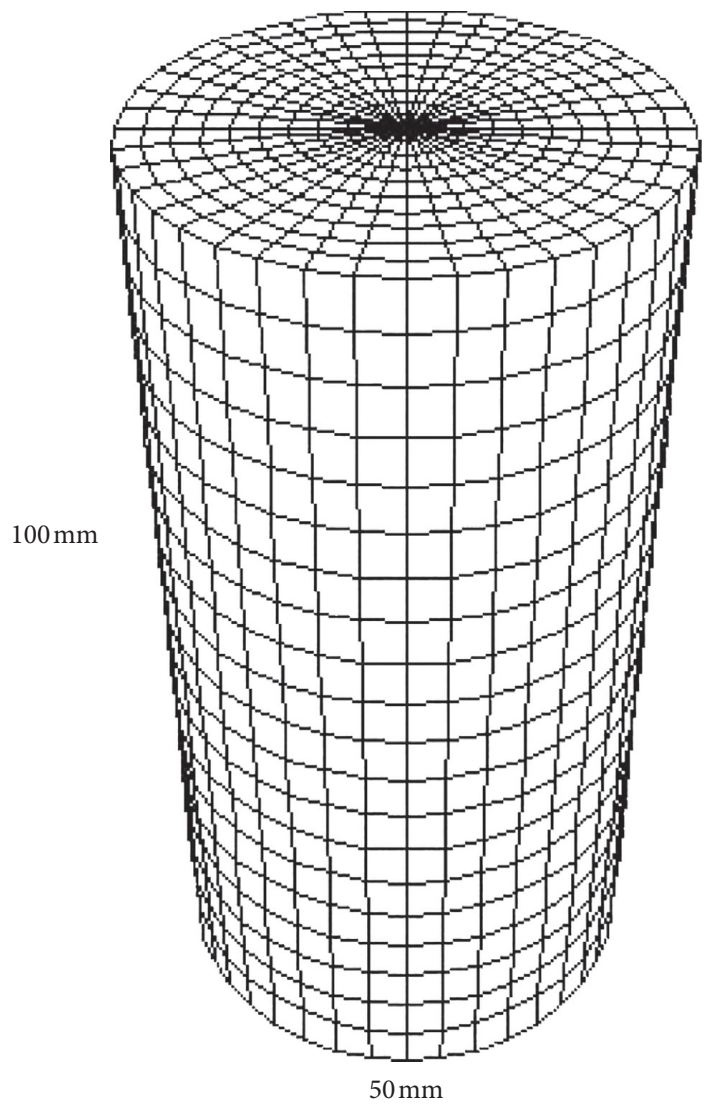

FIGURE 10: Mesh sketch of calculation model.

TABLE 1: Model parameter identification results.

\begin{tabular}{lccccrrr}
\hline Deviatoric stress $(\mathrm{MPa})$ & $E_{0}(\mathrm{MPa})$ & $E_{1}(\mathrm{MPa})$ & $\eta_{1}^{\beta}(\mathrm{MPa} \cdot \mathrm{h})$ & $\beta$ & $\eta_{2}^{\gamma}(\mathrm{MPa} \cdot \mathrm{h})$ & $\gamma$ & - \\
\hline 50 & 360.11 & 1143.59 & 55437.6 & 0.525 & - & - & 0.9970 \\
60 & 383.83 & 348.74 & 2509.21 & 0.150 & - & 0.9973 \\
70 & 359.13 & 2277.96 & 48483.76 & 0.605 & 10560.01 & 0.598 \\
75 & 336.51 & 1986.30 & 22215.98 & 0.566 & 8652.58 & 0.9973 \\
77.5 & 302.45 & 671.81 & 7821.49 & 0.445 & 4621.78 & 0.470 & 0.9979 \\
\hline
\end{tabular}

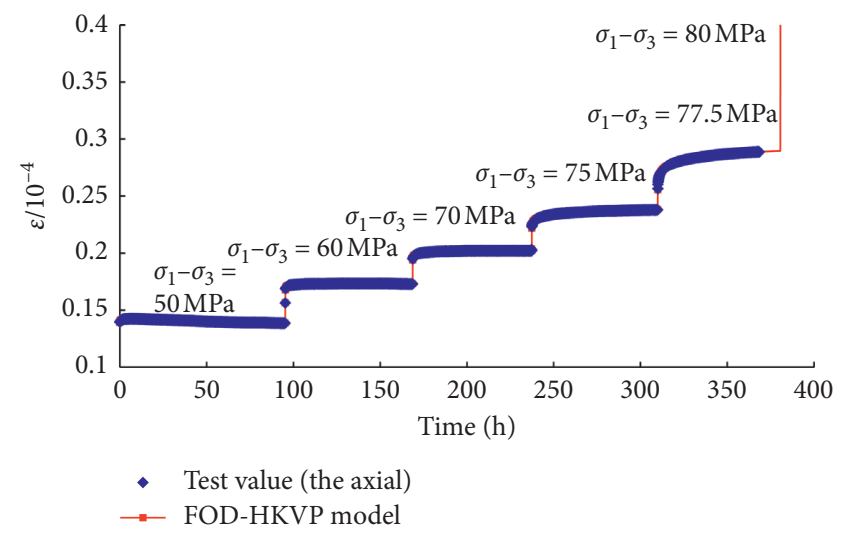

FIGURE 11: Experimental data and the fitting curves for the FODHKVP model.
Analysis of the axial rheological parameter change rule indicates the following:

(1) When the deviatoric stress amounts to $60 \mathrm{MPa}$, parameter volatility is significant. The reason for this is because partial damage occurred within the rock, thereby meaning that it does not arise in the correlation analysis. In addition to this, with an increase in the stress level, the elasticity variable $E(t)$ diminishes gradually, thus fully demonstrating the deterioration effect of hard rock in the unloading creep process.

(2) The viscous variables, $\eta_{1}^{\beta}$ and $\eta_{2}^{\gamma}$, in two viscous bodies decrease gradually in line with rock attenuation creep, steady-state creep, and accelerated creep. This is indicative of the fact that the viscous variable is characterised by its deterioration effect in the context of the unloading rheological process. 


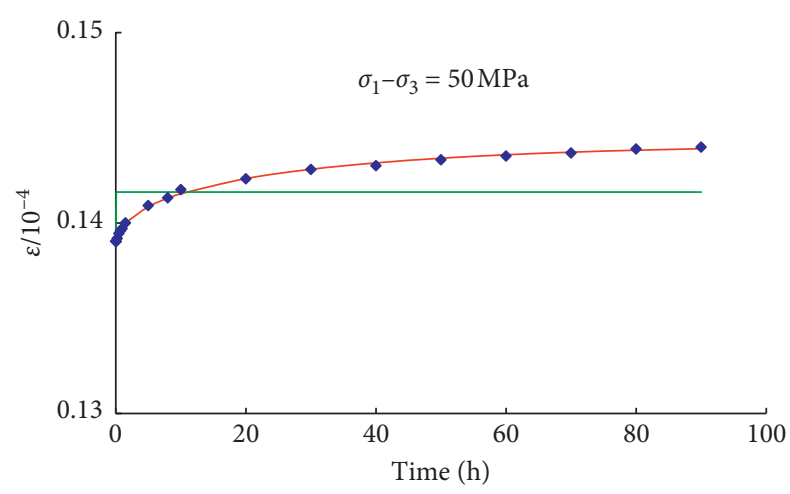

- Test value

— Fitted value of FOD-HKVP model

_ Fitted value of Nishihara model

(a)

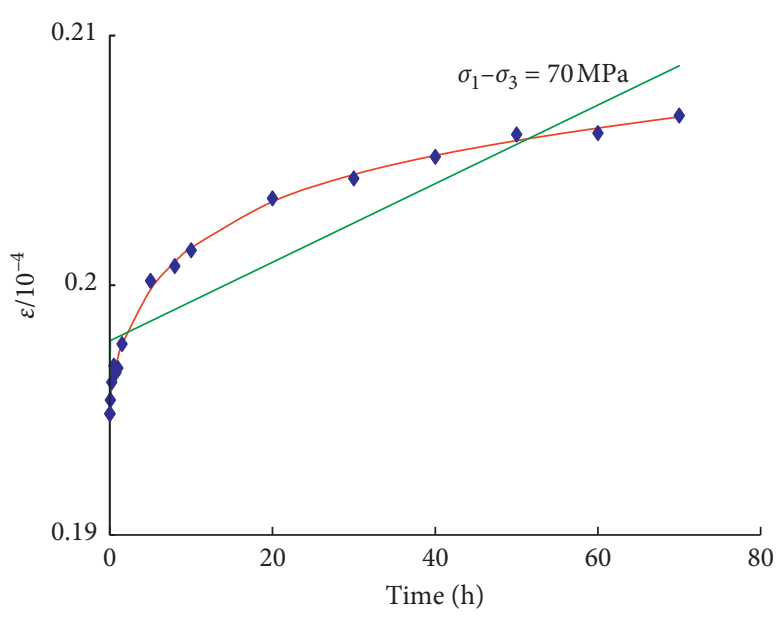

- Test value

— Fitted value of FOD-HKVP model

_ Fitted value of Nishihara model

(c)

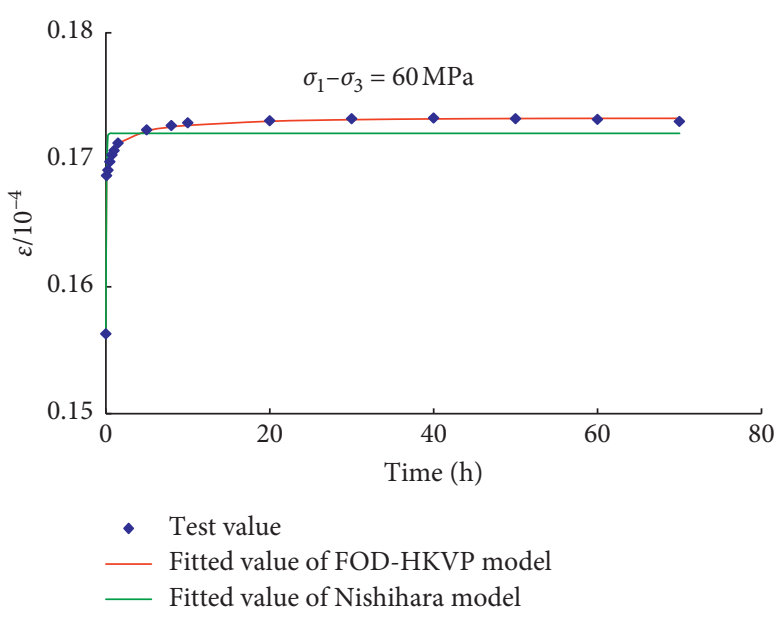

(b)

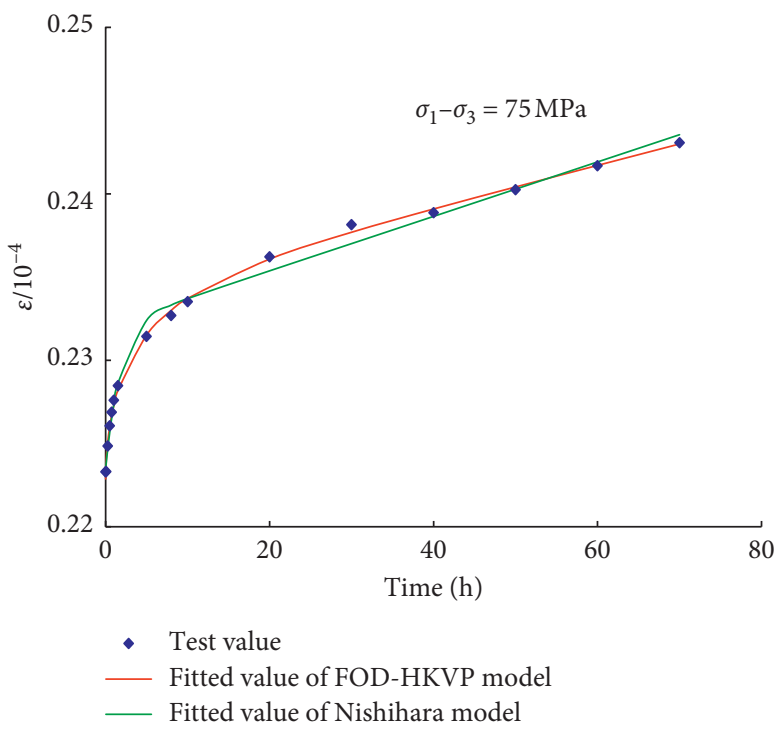

(d)

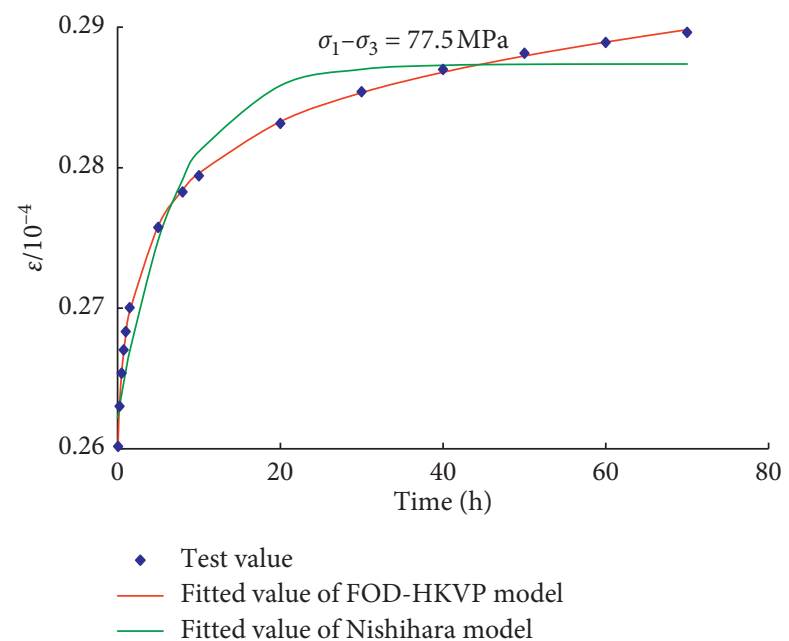

(e)

FIgURE 12: Comparison diagram of fitting results with FOD-HKVP model and the classical Nishihara model. (a) $\sigma_{1}-\sigma_{3}=50 \mathrm{MPa}$. (b) $\sigma_{1}-\sigma_{3}=60 \mathrm{MPa}$. (c) $\sigma_{1}-\sigma_{3}=70 \mathrm{MPa}$. (d) $\sigma_{1}-\sigma_{3}=75 \mathrm{MPa}$. (e) $\sigma_{1}-\sigma_{3}=77.5 \mathrm{MPa}$. 
(3) The fractional derivatives, $\beta$ and $\gamma$, in two viscous bodies are different. In particular, when the stress level exceeds the long-term strength, the fractional derivative decreases with an increase in the stress level, and in the context of the steady-state creep stage, $\beta$ is greater than $\gamma$. However, following the transition from the steady-state creep stage into the accelerated creep stage, $\gamma$ becomes greater than $\beta$. In theory, this is because the two types of viscous bodies describe different stages of the creep process, and the classification of viscous bodies in the fractional-order viscous plastic bodies accounts for the accelerated creep stage. As such, its derivative order, $\gamma$, is greater than the derivative order of the viscous coefficient for the viscoelastic body, $\beta$.

Using the parameter identification results for FODHKVP model verification, Figure 11 compares the theoretical curve against the test curve. Based on the model validation results given in the figure, it is not difficult to see that the theoretical curve closely aligns with the test curve, thus showing that the FOD-HKVP model reflects the unloading creep property of each phase in terms of the hard rock unloading rheological characteristics. As such, the FOD-HKVP model test and theoretical analysis are a close match.

\section{Comparison with the Nishihara Model}

We know from the previous analysis that the classical Nishihara model is a particular instance of the FOD-HKVP model. So in order to further verify the applicability of FODHKVP model, typical test values of each creep stage of rock samples were selected and then fitted with FOD-HKVP model and the classical Nishihara model, respectively, and the fitting results of the two models were compared and analyzed. Figure 12 shows the fitted results.

As it can be seen from the fitting curve in Figure 12, the fitting result of the Nishihara model deviates greatly from the test value, while the FOD-HKVP model can better match the test data and can reflect the whole unloading creep process of granite. Granite belongs to hard rock material, at the initial confining pressure ( $40 \mathrm{MPa}$ ) unloading conditions, when the stress level exceeds its long-term strength value $(62.6 \mathrm{MPa})$, the plastic deformation effects significantly; particularly under the higher horizontal stress, the granite rock turns into the viscoplastic deformation. Compared with the status of viscoelastic deformation with lower stress, the Nishihara model can better describe the relaxation properties of rock, which is reflected in Figure 12; that is, when the stress level is more than $70 \mathrm{MPa}$, the Nishihara model presents a better fitting status. However, compared with the FOD-HKVP model, the fitting degree is still defective. It can be seen from the comparison between the fitting value and the experimental value of the two models that the FOD-HKVP model occupies the advantage. This is because the fractional viscosity coefficient introduced in the FOD-HKVP model can better reflect the viscoelastic deformation at low stress level and the viscoplastic deformation at high stress level.

\section{Conclusion}

The purpose of this research has been to study the unloading rheological properties of hard rock and draw on the theory of fractional-order calculus, as well as damage theory, to construct a nonlinear viscous elastic and plastic damage rheological model, which is called FOD-HKVP model herein. In addition, a comparative analysis was made between the FOD-HKVP model and the classical Nishihara model. The conclusions are as follows:

(1) Relying on the background project of mengjigou hydropower station, the unloading rheological test of the granite under confining pressure of $40 \mathrm{MPa}$ was completed according to the test programme.

(2) Based on the unloading rheological damage characteristics of granite and the analysis of rheological stress-strain curve, the macroscopic and microscopic failure modes, the research sought to illuminate the entire unloading creep process of hard rock.

(3) The FOD-HKVP model was built by using the curve variation characteristics of unloading creep test of granite, and experimental verification proceeded on the basis of the unloading rheological model. It is worth acknowledging that the model identification result is reasonable, and in particular, it is consistent with the unloading creep rule of granite, and the results indicated a close match between the FODHKVP model test and theoretical analysis.

(4) The FOD-HKVP model was compared with the Nishihara model; the results indicated that the FOD-HKVP model can effectively describe the three unloading creep stages for granite. When the stress level is lower, below the long-term strength, the Nishihara model cannot simulate the viscoelastic deformation of granite, while the FODHKVP model presents a perfect fitting curve. When the stress level is higher, above the long-term strength, the Nishihara model fitting has been improved to reflect the viscoplastic creep characteristics of granite, but the accuracy is not good, while the FOD-HKVP model still presents a perfect fitting curve. That is to say, the FOD-HKVP model has obvious advantages in the whole creep process, which relate primarily to the timely adjustment of the viscous coefficient and its fractional derivative order, as well as its description of the nonlinear change process that extends from the steady-state creep stage to the accelerated creep stage. Noteworthily, the model provides an accurate reflection of unloading creep damage characteristics for the hard rock at various stages.

\section{Data Availability}

Some of the experimental test data used to support the findings of this study are included within the article. The other data used to support the findings of this study are available from the corresponding author upon request. 


\section{Conflicts of Interest}

The authors declare that they have no conflicts of interest regarding the publication of this paper.

\section{Acknowledgments}

This work was supported by the National Natural Science Foundation of China (no. 51809156) and the Key Laboratory of Large Structure Health Monitoring and Control, Shijiazhuang (no. KLLSHMC1914).

\section{References}

[1] Y. Wu and L. Jiding, "Unloading properties of marble," Rock and Soil Mechanics, vol. 5, no. 1, pp. 30-36, 1984.

[2] T. Li and W. Lansheng, "An experimental study on the deformation and failure features of a basalt under unloading condition," Chinese Journal of Rock Mechanics and Engineering, vol. 12, no. 4, pp. 321-327, 1993.

[3] J. Shen, L. Wang, and Q. Wang, "Deformation and fracture features of unloaded rock mass," Chinese Journal of Rock Mechanics and Engineering, vol. 22, no. 12, pp. 2028-2031, 2003.

[4] J. S. O. Lau and N. A. Chandler, "Innovative laboratory testing," International Journal of Rock Mechanics and Mining Sciences, vol. 41, no. 8, pp. 1427-1445, 2004.

[5] S. Sorace, "Effects of initial creep conditions and temporary unloading on the long-term response of stones," Materials and Structures, vol. 31, no. 212, pp. 555-562, 1998.

[6] A. A. Bazhin and E. V. Murashkin, "Creep and stress relaxation in the vicinity of a micropore under the conditions of hydrostatic loading and unloading," Doklady Physics, vol. 57, no. 8, pp. 294-296, 2012.

[7] Y. Zhao, L. Zhang, W. Wang et al., "Creep behavior of intact and cracked limestone under multi-level loading and unloading cycles," Rock Mechanics and Rock Engineering, vol. 50, no. 6, pp. 1409-1424, 2017.

[8] D.-Y. Jiang, J.-Y. Fan, and J. Chen, "Study of dilatancy characteristics of salt rock under unloading action of confining pressure," Rock and Soil Mechanics, vol. 34, no. 7, pp. 1881-1886, 2013.

[9] J. Liu, L. Wang, and J.-L. Pei, "Experimental study on creep deformation and long-term strength of unloading-fractured marble," European Journal of Environmental and Civil Engineering, vol. 19, pp. 97-107, 2015.

[10] H.-F. Deng, M.-L. Zhou, and L. I. Jian, "Experimental research on unloading triaxial rheological mechanical properties of sandy mudstone," Rock and Soil Mechanics, vol. 37, no. 2, pp. 315-322, 2016.

[11] Y. Wang, Q. Qiao, and J. Li, “The effect of initial creep damage on unloading failure properties of sandstone from macromesoscopic perspective," Periodica Polytechnica-Civil Engineering, vol. 63, no. 4, pp. 1004-1015, 2016.

[12] H. W. Zhou, C. P. Wang, B. B. Han, and Z. Q. Duan, “A creep constitutive model for salt rock based on fractional derivatives," International Journal of Rock Mechanics and Mining Sciences, vol. 48, no. 1, pp. 116-121, 2011.

[13] Y. Wang, J. Li, and H. Deng, "Investigation on unloading triaxial rheological mechanical properties of soft rock and its constitutive model," Rock and Soil Mechanics, vol. 33, no. 1, pp. 3338-3344, 2012.

[14] B. Nedjar and R. L. Roy, "An approach to the modeling of viscoelastic damage. Application to the long-term creep of gypsum rock materials," International Journal for Numerical and Analytical Methods in Geomechanics, vol. 37, no. 9, pp. 1066-1078, 2013.

[15] W. Chen and H. Konietzky, "Simulation of heterogeneity, creep, damage and lifetime for loaded brittle rocks," Tectonophysics, vol. 633, pp. 164-175, 2014.

[16] Y. Lu, D. Elsworth, and L. Wang, "A dual-scale approach to model time-dependent deformation, creep and fracturing of brittle rocks," Computers and Geotechnics, vol. 60, pp. 61-76, 2014.

[17] F. Wu, J. F. Liu, and J. Wang, "An improved Maxwell creep model for rock based on variable-order fractional derivatives," Environmental Earth Sciences, vol. 73, no. 11, pp. 6965-6971, 2015.

[18] X. Li and Z. Shao, "Investigation of macroscopic brittle creep failure caused by microcrack growth under step loading and unloading in rocks," Rock Mechanics and Rock Engineering, vol. 49, no. 7, pp. 2581-2593, 2016.

[19] P. A. L. P. Firme, D. Roehl, and C. Romanel, "An assessment of the creep behaviour of Brazilian salt rocks using the multimechanism deformation model," Acta Geotechnica, vol. 11, no. 6, pp. 1445-1463, 2016.

[20] H. Tang, D. Wang, R. Huang, X. Pei, and W. Chen, “A new rock creep model based on variable-order fractional derivatives and continuum damage mechanics," Bulletin of Engineering Geology and the Environment, vol. 77, no. 1, pp. 375-383, 2018.

[21] K. Hu, F. Qian, H. Li, and Q. Hu, "Study on creep characteristics and constitutive model for thalam rock mass with fracture in tunnel," Geotechnical and Geological Engineering, vol. 36, pp. 827-834, 2018.

[22] R. Wang, Y. Jiang, C. Yang, F. Huang, and C. Wang, "A nonlinear creep damage model of layered rock under unloading condition," Mathematical Problems in Engineering, vol. 2018, Article ID 8294390, 8 pages, 2018.

[23] K. Balasubramaniam, J. S. Valluri, and R. V. Prakash, "Creep damage characterization using a low amplitude nonlinear ultrasonic technique," Materials Characterization, vol. 62, no. 3, pp. 275-286, 2011.

[24] Z. Hou, "Mechanical and hydraulic behavior of rock salt in the excavation disturbed zone around underground facilities," International Journal of Rock Mechanics and Mining Sciences, vol. 40, no. 5, pp. 725-738, 2003.

[25] Z. He, Z. Zhu, and M. Zhu, "An unsteady creep constitutive model based on fractional order derivatives," Rock and Soil Mechanics, vol. 37, no. 3, pp. 737-744, 2016. 